\title{
Comparison of SAR Data and Operational Sea Ice Products to EM Ice Thickness Measurements in the Baltic Sea
}

\author{
Juha Karvonen ${ }^{1}$, Markku Similä ${ }^{1}$, Jari Haapala ${ }^{1}$ \\ Christian Haas ${ }^{2}$, Marko Mäkynen ${ }^{3}$ \\ ${ }^{1}$ Finnish Institute of Marine Research (FIMR), PB 33, FIN-00931, Helsinki, Finland \\ Ph. +358 961394 424, Fax +3589 3231025, Email: Juha.Karvonen@ fimr.fi \\ 2 Alfred Wegener Institute (AWI), Bremerhaven, Germany \\ ${ }^{3}$ Helsinki Univ. of Technology, Laboratory of Space Technology (HUT/LST), Finland
}

\section{INTRODUCTION}

In February 2003, sea ice thickness measurements using an electromagnetic induction (EM) instrument were made in the Gulf of Bothnia and Gulf of Finland. We have made comparisons between the EM measurements and Radarsat1 ScanSAR Wide mode SAR data, and also between our operational sea ice products (digitized ice thickness charts, and ice thickness charts refined by the latest Radarsat- 1 image). The SAR images are in $100 \mathrm{~m}$ resolution, and the other products are in $500 \mathrm{~m}$ resolution.

The maximum daily temperatures during the EM measurement campaign were typically above zero degrees, probably making the ice surface and snow on the ice wet in the daytime, and thus attenuating the SAR backscattering from the sea ice. This data set mainly describes the statistics of wet snow or frozen snow-surface conditions, and a similar study for dry snow conditions would also be useful. The time gap between SAR image and the EM measurement varied from about 2 hours to about 9.5 hours, and the wind speeds between the SAR acquisition and EM measurement were relatively low (in maximum about $4 \mathrm{~m} / \mathrm{s}$ in the coastal stations).

\section{Measuring The ICE Thickness By ELECTROMAGNETIC INDUCTION}

Alfred Wegener Institute (AWI) has performed EM measurements in Gulf of Bothnia and Gulf of Finland in 2003 and 2004. The helicopter-borne EM instrument measures the distance to the water below sea ice based on low-frequency electromagnetic induction from the sea water. Sea ice is typically resistive and water is conductive and electromagnetic induction from the water occurs. The ratio between secondary and primary fields, transmitted and received/measured by the EM instrument, depends on the distance between the EM system and the conductive media, and on the electrical conductivity of the media. Main part of the secondary magnetic field comes from the sea water, and the instrument distance to the sea water can be derived from the measured secondary field strength. Then the ice (plus snow) thickness is the distance to the sea water, measured by the EM instrument, subtracted by the distance to the surface measured by a laser altimeter included in the instrument. The EM measurement principle is shown in Fig. 1.

It has been assessed that for level ice the accuracy of EM measurements is about $10 \mathrm{~cm}$ [1] [2] [3]. For ridged ice the

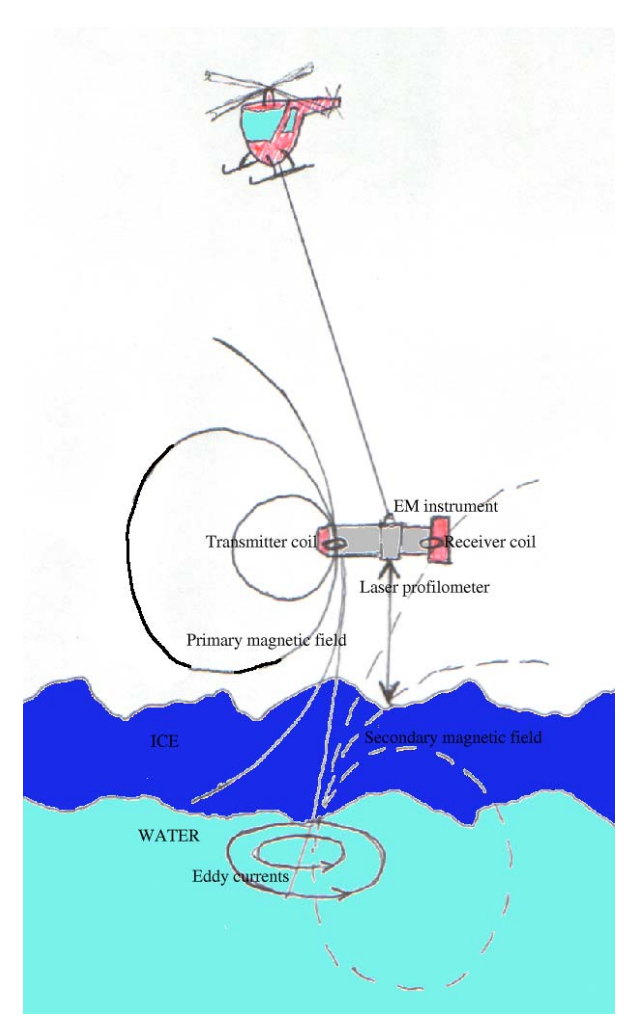

Fig. 1. The EM measurement principle.

accuracy is weaker due to water appearing between ice blocks. However, almost always the occurrence of an ice ridge can be detected.

\section{SAR DATA PREPROCESSING}

We have compared Radarsat-1 ScanSAR Wide mode SAR image data pixel values values and our operational sea ice products of the corresponding days with the EM measurements. The operational products are the ice thickness chart, derived from the digitized ice chart and the ice thickness chart refined by the information of the most recent SAR data [4].

The SAR data is received in a logarithmic scale from Kongsberg Satellite Services (KSS) in Tromsö, Norway. The relation between the measured SAR intensity $I$ and the pixel value $P$ in our images is

$$
I=\left(\frac{B^{P}}{G}\right)^{2}
$$


where $\mathrm{P}$ is the 8-bit image pixel value, logarithmic scale $B=$ 1.024 , and gain factor $G=0.16$. The data has this far not been absolutely calibrated.

The data are in different resolutions, the sampling rate of the EM measurement is 3-4 m and the EM measurement resolution is around $20-30 \mathrm{~m}$, and the Radarsat- 1 images are in $100 \mathrm{~m}$ resolution. The nominal resolution of digitized ice thickness charts is about $1 \mathrm{~km}$, and the resolution of our operative SARrefined ice thickness chart is $500 \mathrm{~m}$. In $100 \mathrm{~m}$ resolution the flight line in maximum covers about $20 \%$ of the pixel area (assuming straight flight line over the pixel center), and in 500 $\mathrm{m}$ resolution this area is only $4 \%$. The only reasonable way to compare these kind of data is to make statistical comparisons. The material covers five SAR images from 4 days, and about $1000 \mathrm{~km}$ of EM flight lines in total. The measurements were conducted in highly ridged drift ice area between February 17th and February 23rd 2003 in Gulf of Finland and Gulf of Bothnia.
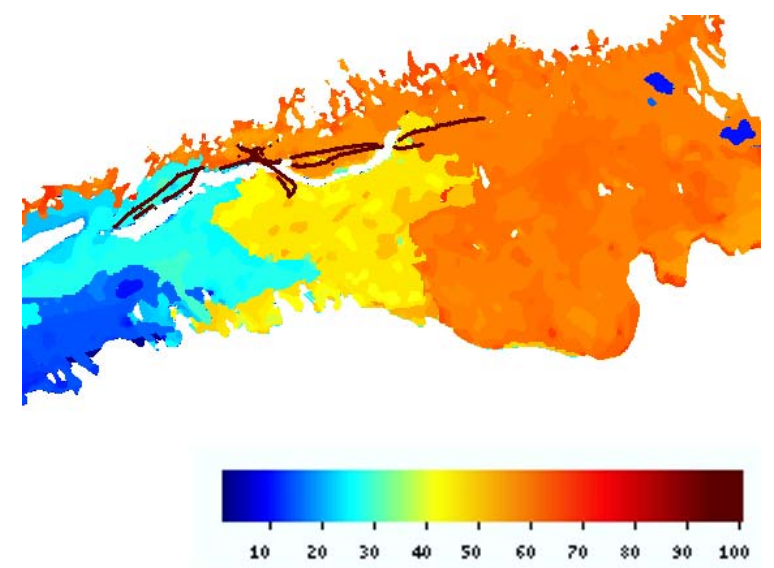

Fig. 2. Flight lines on Feb 17th 2003 drawn (dark lines) on a SAR-refined ice thickness map, Gulf of Finland. The scale in the figure is in $\mathrm{cm}$.

Before the comparisons an incidence angle correction designed for Baltic Sea ice [5] [6] was applied to the SAR data, normalizing the SAR pixel values to correspond to an incidence angle of 35 degrees (in the middle of the Radarsat-1 ScanSAR Wide mode incidence angle range).

For each SAR and product pixel in the EM profiles a distribution of the EM values was computed, and the values derived from these distributions were used in the comparisons.

\section{EXPERIMENTAL RESUlts}

To analyze our data, we coarsely divided the sea ice, based on the EM ice thickness measurements, into three categories, one representing level ice corresponding to thermal growth, the other rafted ice, and the third representing ridged ice. Additionally there also exists open water. Level formed by thermal growth in our scheme, is defined as ice with EM thickness less than $50 \mathrm{~cm}$ (representing the estimated maximal thermal growth until late February, being about 50-60 cm), rafted ice is derfined as ice of EM thickness range from 50 to $100 \mathrm{~cm}$ (corresponding to doubles of the level ice thicknesses), and ridged ice is defined as ice with EM thickness over 100 $\mathrm{cm}$ (including higher multiples of the level ice).

In our statistical analysis, the most significant statistical relationship between the incidence angle corrected SAR pixel values and the EM thickness distribution was established as follows. The range of the corrected SAR pixel values over their dynamic value range was first divided into 15 equal-sized bins. Then, given a fixed bin, we computed the conditional distribution of the three ice thickness categories based on the EM thickness values. It was observed that the fraction of small ridges (rafted ice) remained relatively constant independent of the SAR pixel value. In this data set with these thickness limits, this fraction remained at about $30 \%$. On the other hand, the area covered by large ridges grows almost linearly from 0 $10 \%$ at very low amplitude values to $90-100 \%$ at the highest amplitude values. The fraction of level ice decreased from 70 $\%$ at low amplitudes to less than $10 \%$ at high amplitude values, see Fig. 3. If the limits in the three ice thickness categories are changed the figures of relative fraction change also but qualitatively they exhibit the same kind of behavior.

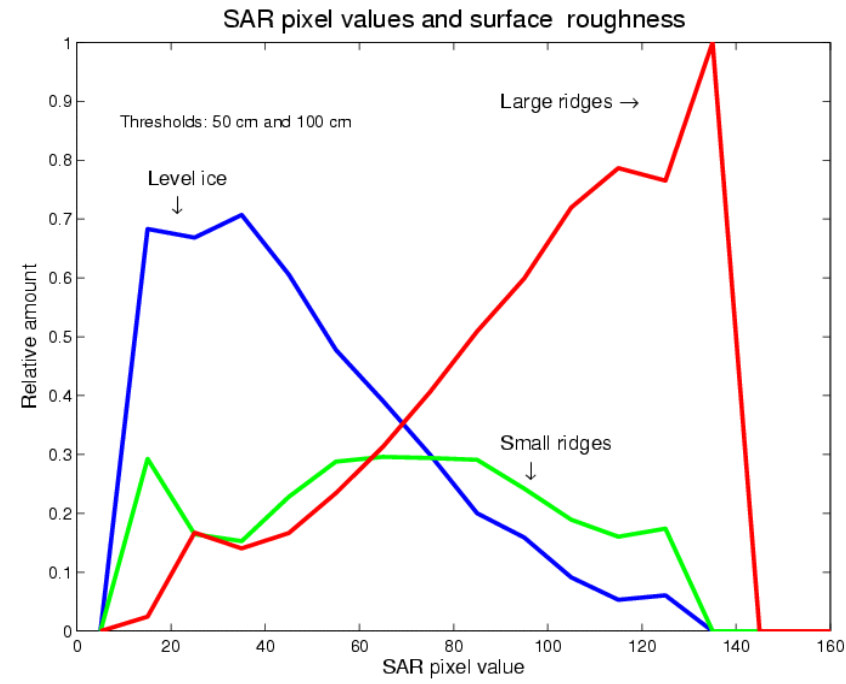

Fig. 3. The relative amounts of the three different ice types as a function of incidence angle corrected SAR intensity.

We also examined the (cross-)correlations between the incidence angle corrected SAR pixel values, ice thickness of the ice charts, the SAR enhanced ice thickness charts and values computed from the EM measurements. For each SAR or product pixel, we first determined a distribution of the measured EM thicknesses. From this histogram we computed the relative amounts of the three ice classes, the mean thickness and the thickness mode over each $500 \mathrm{~m}$ pixel. In a $500 \mathrm{~m}$ pixel the number of EM measurements in one pixel was in maximum about 130 measurements, but this naturally for the case in which the flight line goes through the midpoint of the pixel. Actually, only the distributions where the number of EM measurements exceeded a threshold value (20) were used in the computations. The correlations are shown in Table I.

The trends seen in these correlations are consistent with the results presented in Fig. 3. The correlation between the SAR 
TABLE I

CORRELATION COEFFICIENTS BETWEEN THE PRODUCTS AND PIXEL-WISE VALUES DERIVED FROM THE EM MEASUREMENTS. THE PIXEL RESOLUTION IS 500M.

\begin{tabular}{|c|c|c|c|}
\hline Variable & SAR & Thickn. & Refined thickn. \\
\hline level & -0.32 & -0.18 & -0.21 \\
rafted & 0.02 & 0.16 & 0.12 \\
ridged & 0.30 & 0.07 & 0.12 \\
mean & 0.25 & 0.11 & 0.14 \\
mode & 0.16 & 0.14 & 0.20 \\
\hline
\end{tabular}

pixel value and the relative amount of level ice is negative, the correlation between the SAR pixel value and the relative amount of rafted ice is practically zero, and the correlation between the SAR pixel value and the relative amount of ridged ice is positive. Also it can be seen that the correlations between the EM measurement values describing the thickness (mean, mode) and the refined thickness chart are slightly higher than the corresponding values for the ice chart.

The digitized ice thickness chart has three thickness values for each segment, i.e. the level ice minimum, mean and maximum thicknesses. In our data along the flight lines, we could find the following different classes described by the triplet thickness minimum-mean-maximum: 5-5-5, 5-5-10, 5$15-30,10-15-30,10-20-30,20-25-40,20-30-50,20-35-50,10-$ $40-50,30-40-60,30-45-60$ and 40-50-70.

The accuracy of the estimates given by different ice thickness charts is assessed as follows. First, the digitized ice charts are divided into bins with a varying bin width in the order described above. The SAR refined ice charts are divided into 30 bins with a fixed bin width of $2 \mathrm{~cm}$. Secondly, the classwise ice chart distributions for open water and the three ice types are calculated for each bin. In the ideal case, the amount of deformed ice types increases with the estimated level ice thickness (it is probable that older ice has gone through more deformations than younger ice). For the digitized ice charts this seems not to be the case. There occurs random-like fluctuation in the fraction of highly ridged ice areas up to the estimated mean level of about $35 \mathrm{~cm}$. After that the amount of highly deformed ice area remains high and, approximately, at the same level, i.e., the higher bins are not significantly different from each other. For details see fig. 4 upper panel.

For the SAR refined thickness chart, the results are more satisfactory. Excluding the thin ice case (estimated thickness less than $4 \mathrm{~cm}$ ), the amount of deformed ice types increases as a function of the level ice thickness estimate until about 35 $\mathrm{cm}$. Then a state is reached where the fraction of deformed ice type remains approximately at the same level independent of the thickness estimate. This is explicable, because the initial data (ice chart thickness) has this same deficiency and in the forming of SAR refined thickness chart some very restrictive rules are applied [4]. These restrictive rules are necessary, because on the basis of single (speckled) pixel value one can not make any confident statement about the degree of ice deformation, see Table I. For details see fig. 4 lower panel.

In the areas identified to represent very thin ice, 1 to $4 \mathrm{~cm}$, by the SAR refined ice chart, there occurred also relatively high fractions of deformed ice type (fig. 4). To explain this peculiar behavior, we checked the location of measurements where the SAR-refined ice chart thickness is less than 10 $\mathrm{cm}$. More than half of the EM thickness measurements inside the pixel have thicknesses of more than $50 \mathrm{~cm}$. We found that these measurements typically were made at the edges of thin ice or leads (open water areas), according to the SAR-refined ice chart. These erroneous pixels also typically appear in relatively short segments. Such errors are probably due to the time differences between the SAR images and EM measurements, it is probable that sea ice movement and reformation have taken place between the SAR and EM measurements. Probably a larger amount of EM measurements over areas with thin ice and even larger open water areas, not just narrow leads, would have yielded more accurate results for the thin ice areas (according to the SAR-refined ice charts).
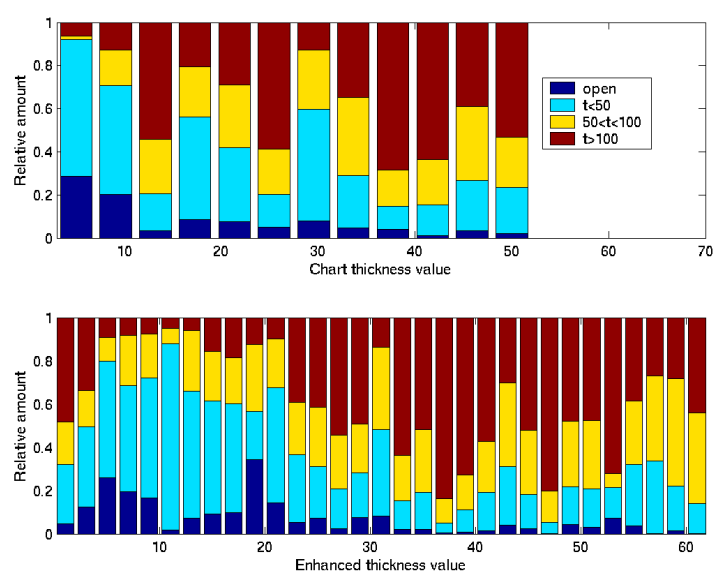

Fig. 4. The relative amounts of the three ice types and open water for the ice types present in the ice chart (upper figure), and for the refined ice chart(lower figure).

\section{CONCLUSION AND DISCUSSION}

Only weak correspondence was observed when comparing the data sets pixel by pixel, i.e., the correlations between the SAR data or the operational products and the EM measurements are relatively low. This ambiguity is partly due to the ice movement between the SAR data acquisition and EM measurements, and possible inaccuracies in registration between the two data sets. One has, however, to keep in mind that quite different ice conditions can give a similar backscattering signature [7]. However, by examining a large data set, clear statistical relationships between ice types and the strength of backscattering could be detected.

On the basis of our data sets, it seems that at $100 \mathrm{~m}$ resolution the SAR data does not improve our a priori knowledge about the area covered by small ridges in the Baltic Sea. However, even at this coarse resolution the backscattering level correponds quite well with the amount of deformed ice consisting of large ridges. We recall that the measurements were mostly made in highly ridged drift ice. Hence, the last conclusion is valid only for this kind of ice conditions. 
The earlier comparisons, e.g. in [8], show that the ice thickness typically changes as the SAR mean intensity or texture changes, and EM measurements can be used as a method to include more precise thickness information into the SAR segments. Our experiments agree with this conclusion, and it seems that, based on a sample of SAR backscattering intensity values, it is possible to assign a crude estimate to the ice type distribution for the ice field from which this scattering originates. This assignment requires, however, a priori knowledge about the order of ice thickness. This information is provided by the initial datum of the algorithm.

EM profiling is a good tool for verifying our operational sea ice products. The SAR-refined ice chart seems to indicate better the local mean ice thickness than the ice chart, based on comparison to the EM measurements. The EM measurement statistics can also be used to make some algorithm improvements.

There are some restrictions in the EM measurements: the size of the instrument footprint is about 20-30 meters, i.e. measurements average over an area of this diameter. Also signals from fresh water, e.g., near river outlets, shallow water, no water at all, can cause misinterpretations. The calibration was performed for each flight line separately, assuming open water at the minimum, and in some cases this leads to underestimation (bias) by the minimum level ice thickness in the line (no open water).

In February-March 2004 several EM measurement flights were made in the Gulf of Bothnia. The data will be utilized in future comparisons. We also have high-resolution Envisat ASAR IMP and APP mode data acquired during this period, making statistical comparisons in more detail possible.

\section{REFERENCES}

[1] C. Haas, W. Dierking, M. Lensu, Airborne Measurements of Baltic Ice Thickness in February 2003: The Campaign, Report of the EU-project IRIS (EVK3-CT-2002-00833), 2003.

[2] C. Haas, IRIS Data Report: Airborne EM Measurements of Baltic Ice Thickness in February 2003, Report of the EU-project IRIS (EVK3-CT2002-00833), 2003.

[3] C. Haas, Airborne EM Sea-Ice Thickness Profiling over Brackish Baltic Sea Water, in Proc. of the International Symposium on Ice St. Petersburg, 2004.

[4] J. Karvonen, M. Similä, I. Heiler, Ice Thickness Estimation Using SAR Data and Ice Thickness History, Proceedings of the IEEE International Geoscience and Remote Sensing Symposium 2003 (IGARSS'03), v. I, pp. 74-76, 2003.

[5] M. Mäkynen, T. Manninen, M. Similä, J. Karvonen, M. Hallikainen, Incidence Angle Dependence of the Statistical Properties of the C-Band HH-Polarization Backscattering Signatures of the Baltic Sea Ice, IEEE Transactions on Geoscience and Remote Sensing, vol 40, n 12, 2002.

[6] J. Karvonen, M. Similä and M. Mäkynen, An Iterative Incidence Angle Normalization Algorithm for Sea Ice SAR Images, Proceedings of the IEEE International Geoscience and Remote Sensing Symposium 2002 (IGARSS'02), 2002.

[7] M. Mäkynen, M. Hallikainen, Investigation of C-Band and X-Band Backscattering Signatures of the Baltic Sea Ice, International Journal of Remote Sensing, v. 25, n. 11, pp. 2061-2086, 2004.

[8] I. K. Peterson, S. J. Princenberg, S. Holladay, Mapping Sea Ice Thickness with RADARSAT and a Helicopter-Borne Electromagnetic-Induction System, in Proc. ERS-Envisat Symposium "Looking down to Earth in the New Millennium" SP-461, 16-20 October Gothenburg, Sweden, ESA, CD-ROM, 2000. 